\title{
MicroRNA-22 is downregulated in clear cell renal cell carcinoma, and inhibits cell growth, migration and invasion by targeting PTEN
}

\author{
WENXING FAN $^{1 *}$, JIE HUANG $^{1 *}$, HUA XIAO ${ }^{1}$ and ZHANG LIANG ${ }^{2}$ \\ ${ }^{1}$ Department of Nephrology, The First Affiliated Hospital of Kunming Medical University, Kunming, Yunnan 650032; \\ ${ }^{2}$ Department of Science and Technology, Kunming Medical University, Kunming, Yunnan 650500, P.R. China
}

Received May 6, 2015; Accepted March 3, 2016

DOI: $10.3892 / \mathrm{mmr} .2016 .5101$

\begin{abstract}
MicroRNA (miR)-22 has previously been reported to be frequently downregulated in certain types of cancer. The present study examined the expression and effects of miR-22 in renal cell carcinoma (RCC). The results indicated that miR-22 was downregulated in tumor tissue from patients with RCC. In addition, lower miR-22 expression levels were associated with histological grade, tumor stage and lymph node metastasis. Following transfection of RCC cells with miR-22, 3-(4,5-dimethylthiazol-2-yl)-2,5-diphenyltetrazolium bromide, cell migration, cell invasion and luciferase assays, and western blotting were conducted. The results demonstrated that miR-22 was able to inhibit cell proliferation, migration and invasion in 786-O and A498 cells. Furthermore, the results indicated that miR-22 may directly target phosphatase and tensin homolog (PTEN) in RCC. In conclusion, the present study suggested that the miR-22/PTEN axis may be considered a novel therapeutic target in RCC. These findings may be beneficial for the development of an effective therapy against RCC.
\end{abstract}

\section{Introduction}

Renal cell carcinoma ( $\mathrm{RCC}$ ) is one of the most common types of urological cancer, which accounts for 2-3\% of all cancers in adults. The incidence of RCC is ranked seventh in men and ninth in women (1). Worldwide, there are 209,000 newly diagnosed cases of RCC and $\sim 102,000$ cases of RCC-associated

Correspondence to: Professor Hua Xiao, Department of Nephrology, The First Affiliated Hospital of Kunming Medical University, 295 Xichang Road, Kunming, Yunnan 650032, P.R. China

E-mail: huaxiao88966@163.com

${ }^{*}$ Contributed equally

Key words: microRNA-22, phosphatase and tensin homolog, renal cell carcinoma mortality per year (2). Among the five subtypes, clear cell RCC (ccRCC), which originates in the lining of the proximal renal tubule, accounts for $\sim 70 \%$ of RCC cases, and is associated with the highest rates of local invasion, metastasis, mortality and resistance to treatment (3). Approximately $70 \%$ of patients present with localized disease, and radical or partial nephrectomy remains the mainstay of curative treatment for RCC (4). However, one third of these patients develop metastatic disease after surgery (5). Patients with metastatic RCC have a poor prognosis and limited therapeutic options, due to the highly resistant phenotype of metastatic RCC to conventional therapeutic modalities, including chemotherapy and radiation. Median survival in a recent cohort of patients with metastatic RCC was 1.5 years, with $<10 \%$ of patients surviving for 5 years (6). Another issue concerning RCC is the absence of specific prognostic biomarkers and follow-up care, thus complicating early diagnosis. These issues make RCC a major challenge in the field of oncology (7); therefore, the identification of novel biomarkers and treatments would be helpful for the management of patients with renal tumors.

MicroRNAs (miRNAs) are a class of small (19-25 nt), endogenous, noncoding RNA molecules, which diversely regulate gene expression at the post-transcriptional level via degradation or translational inhibition of specific target mRNAs $(8,9)$. At present, 1,872 precursors and 2,578 mature miRNAs have been verified in humans (10). It is now evident that miRNAs regulate numerous critical biological processes, including proliferation, differentiation, apoptosis, cell cycle regulation and metastasis (11). miRNAs have been hypothesized to regulate $30 \%$ of protein-encoding genes (12). Furthermore, miRNAs are often reported to be down- or upregulated in human cancers (13). Upregulated miRNAs in cancer may function as oncogenes by negatively regulating the expression of tumor suppressors. Conversely, downregulated miRNAs may normally function as tumor suppressor genes, and inhibit cancer by regulating oncogenes (14). A single miRNA can target several mRNAs simultaneously; therefore, miRNAs have been suggested as a potential method to concurrently modify the activities of numerous pathways. If suitable endogenous or exogenous sequences could be identified, miRNAs may comprise a rational novel approach for the treatment of mechanistically complex diseases (15). At present, the 
use of miRNAs as predictive biomarkers or therapeutic targets in $\mathrm{RCC}$ requires further exploration.

The expression of miRNA (miR)-22 has been reported to be downregulated in certain types of cancer (16-20); however, there are currently no studies regarding the expression of miR-22 in RCC. In addition, the relationship between miR-22 expression in RCC and clinicopathological factors has yet to be elucidated. The present study aimed to investigate the expression of miR-122 in RCC, the association between miR-122 in RCC and clinicopathological features of patients, and the function of miR-122 in RCC. The present study demonstrated that miR-22 was downregulated in human RCC tissues, as compared with in normal adjacent tissues (NATs). Statistical analyses indicated that miR-22 expression was significantly associated with histological grade, tumor stage and lymph node metastasis. Furthermore, miR-22 was able to inhibit cell proliferation, migration and invasion by directly targeting phosphatase and tensin homolog (PTEN). These findings may have diagnostic and therapeutic implications, and could be exploited for the future treatment of RCC.

\section{Materials and methods}

Clinical samples. The present study was approved by the Hospital's Protection of Human Subjects Committee of The First Affiliated Hospital of Kunming Medical University (Kunming, China), and informed consent was obtained from all patients. Tumor tissue specimens and NATs were obtained from 68 patients with ccRCC who had undergone surgery at The First Affiliated Hospital of Kunming Medical University. The tissue specimens were subsequently used for reverse transcription-quantitative polymerase chain reaction (RT-qPCR). The patients all underwent radical nephrectomy. The tumor samples were histopathologically diagnosed as ccRCC, and were snap-frozen in liquid nitrogen and stored at $-80^{\circ} \mathrm{C}$ until further use. The clinical characteristics of the patients with ccRCC are presented in Table I.

$R N A$ isolation and RT-qPCR. The tissue specimens were cut into small pieces and manually homogenized in liquid nitrogen with a mortar and pestle. Total RNA was isolated from the tissues using TRIzol ${ }^{\circledR}$ (Invitrogen; Thermo Fisher Scientific, Inc., Waltham, MA, USA), according to the manufacturer's protocol. The concentration and purity of the total RNA was determined using A260/A280 with NanoDrop ND-2000 UV-Vis spectrophotometer (NanoDrop Technologies; Thermo Fisher Scientific, Inc., Wilmington, DE, USA). cDNA was synthesized using the Moloney Murine Leukemia Virus Reverse Transcription system (Promega Corporation, Madison, WI, USA) in a $25 \mu \mathrm{l}$ reaction volume. The temperature protocol was as follows: $95^{\circ} \mathrm{C}$ for $2 \mathrm{~min}$; 20 cycles of $94^{\circ} \mathrm{C}$ for $1 \mathrm{~min}, 55^{\circ} \mathrm{C}$ for $1 \mathrm{~min}$ and $72^{\circ} \mathrm{C}$ for $2 \mathrm{~min}$; and $72^{\circ} \mathrm{C}$ for 5 min. RT-qPCR was performed in an ABI PRISM 7000 Fluorescent Quantitative PCR system (Applied Biosystems; Thermo Fisher Scientific, Inc.) using the reagents of a SYBR Green I mix (Takara Biotechnology Co., Ltd., Dalian, China) in a $20 \mu \mathrm{l}$ reaction volume, according to the manufacturer's protocol. The reaction system contained $10 \mu \mathrm{l} \mathrm{SYBR}$ Green I mix, $2 \mu$ l forward primer, $2 \mu 1$ reverse primer (each obtained from Guangzhou RiboBio Co., Ltd., Guangzhou, China) and
$4 \mu \mathrm{l}$ double distilled water. The thermocycling conditions of the reaction were as follows: $95^{\circ} \mathrm{C}$ for $10 \mathrm{~min}$; and 40 cycles of $95^{\circ} \mathrm{C}$ for $15 \mathrm{sec}$ and $60^{\circ} \mathrm{C}$ for $1 \mathrm{~min}$. Each sample was analyzed in triplicate and the data was normalized to U6 expression. Relative expression fold changes were calculated using the $2^{-\Delta \Delta C q}$ method (21).

Cell lines and culture conditions. The human ccRCC-derived cell lines 786-O and A498 were purchased from the Shanghai Institute of Biochemistry and Cell Biology (Shanghai, China). The 786-O and A498 cells were cultured in Dulbecco's modified Eagle's medium (Gibco; Thermo Fisher Scientific, Inc.) supplemented with $10 \%$ heat-inactivated fetal bovine serum (FBS), $100 \mathrm{U} / \mathrm{ml}$ penicillin and $100 \mathrm{mg} / 1$ streptomycin (all Gibco; Thermo Fisher Scientific, Inc.) at $37^{\circ} \mathrm{C}$ in a humidified atmosphere containing $5 \% \mathrm{CO}_{2}$.

Transfection with miR-22 mimics, negative control (NC) miRNA mimics and a luciferase reporter plasmid. Mature miR-22 mimics, negative control (NC) miRNA mimics and the luciferase reporter plasmid were designed and synthesized by Shanghai GenePharma Co., Ltd. (Shanghai, China). The sequences of the mimics were as follows: miR-22, 5'-AAG CUGCCAGUUGAAGAACUGU-3'; and NC mimics, 5'-UUC UCCGAACGUGUCACGUTT-3'. Cells were seeded into 6-well plates at a confluency of 60-70\%. Following overnight incubation, transient transfection and co-transfection were performed using Lipofectamine 2000 (Invitrogen; Thermo Fisher Scientific, Inc.) for $6 \mathrm{~h}$ at room temperature, according to the manufacturer's protocol.

Cell proliferation assay. Cell proliferation was measured using the 3-(4,5-dimethylthiazol-2-yl)-2,5-diphenyltetrazolium bromide (MTT) assay. The 786-O and A498 cells transfected with miR-22 or NC mimics were seeded into 96-well culture plates at a density of $4 \times 10^{3}$ cells/well. The proliferation assay was performed for 6 days and cell growth was assayed at every $24 \mathrm{~h}$ interval. Briefly, $20 \mu \mathrm{l} \mathrm{MTT}$ (5 mg/ml; Sigma-Aldrich, St. Louis, MO, USA) solution was added to each well, and the plate was incubated at $37^{\circ} \mathrm{C}$ for $4 \mathrm{~h}$. The plates were then spun, and the purple-colored formazan precipitates were dissolved in $200 \mu 1$ dimethyl sulfoxide. Following a further spin at $7^{\circ} \mathrm{C}$ for $15 \mathrm{~min}$, the optical density of each sample was measured at $490 \mathrm{~nm}$ using an automatic multi-well spectrophotometer (Bio-Rad Laboratories, Inc., Hercules, CA, USA). All experiments were performed in triplicate.

Migration and invasion assays. The migratory and invasive ability of the 786-O and A498 cell lines was assessed using a Transwell apparatus containing an $8 \mu \mathrm{m}$-pore polycarbonate membrane Boyden chamber insert (Costar; Corning Incorporated, Corning, NY, USA). A total of $1 \times 10^{5}$ transfected cells (miR-22 and NC mimics) were resuspended in $0.2 \mathrm{ml}$ serum-free RPMI 1640 medium and seeded into the upper chambers of the Transwell apparatus. RPMI 1640 (600 $\mu \mathrm{l}$; Gibco; Thermo Fisher Scientific, Inc.) containing $20 \%$ FBS was added to the lower chamber. The invasion assay was carried out using the same protocol, however the filters of the Transwell chambers were coated with 
Table I. Comparison between microRNA (miR)-22 expression in renal cell carcinoma and clinicopathological features.

\begin{tabular}{|c|c|c|c|c|}
\hline \multirow[b]{2}{*}{ Clinical feature } & \multirow[b]{2}{*}{ Case number } & \multicolumn{2}{|c|}{ miR-22 expression } & \multirow[b]{2}{*}{ P-value } \\
\hline & & High & Low & \\
\hline \multicolumn{5}{|l|}{ Gender } \\
\hline Male & 38 & 23 & 15 & \multirow[t]{2}{*}{0.552} \\
\hline Female & 30 & 16 & 14 & \\
\hline \multicolumn{5}{|l|}{ Age (years) } \\
\hline$<60$ & 45 & 29 & 16 & \multirow[t]{2}{*}{0.95} \\
\hline$>60$ & 23 & 15 & 8 & \\
\hline \multicolumn{5}{|c|}{ Tumor diameter $(\mathrm{cm})$} \\
\hline$<3$ & 42 & 30 & 12 & \multirow[t]{2}{*}{0.14} \\
\hline$>3$ & 26 & 14 & 12 & \\
\hline \multicolumn{5}{|c|}{ Histological grade } \\
\hline I-II & 40 & 31 & 9 & \multirow[t]{2}{*}{0.004} \\
\hline III-IV & 28 & 12 & 16 & \\
\hline \multicolumn{5}{|l|}{ Tumor stage } \\
\hline $\mathrm{T} 1-\mathrm{T} 2$ & 36 & 28 & 8 & \multirow[t]{2}{*}{0.008} \\
\hline T3-T4 & 32 & 15 & 17 & \\
\hline \multicolumn{5}{|c|}{ Lymph node metastasis } \\
\hline Positive & 14 & 2 & 12 & \multirow[t]{2}{*}{0.0001} \\
\hline Negative & 54 & 38 & 16 & \\
\hline
\end{tabular}

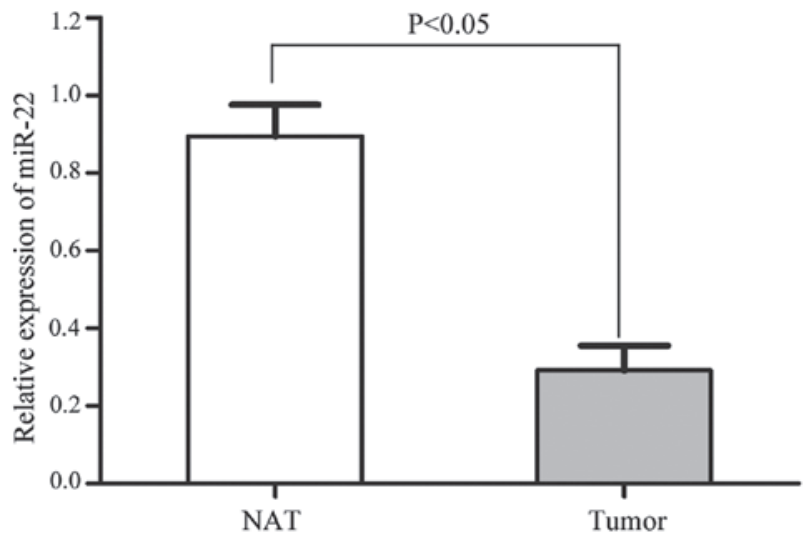

Figure 1. MicroRNA (miR)-22 is downregulated in renal cell carcinoma (RCC). Reverse transcription-quantitative polymerase chain reaction analysis detected a significant downregulation of miR-22 expression in RCC tissues (Tumor) compared with in normal adjacent tissues (NAT). Data are presented as the mean \pm standard deviation.

growth factor-reduced Matrigel (BD Biosciences, San Jose, CA, USA). The cells were incubated at $37^{\circ} \mathrm{C}$ in a $5 \% \mathrm{CO}_{2}$ incubator for $12 \mathrm{~h}$ for the migration assay and $24 \mathrm{~h}$ for the invasion assay. After the cells had been incubated for $12-24 \mathrm{~h}$ at $37^{\circ} \mathrm{C}$ in a $5 \% \mathrm{CO}_{2}$ incubator, they were fixed with $10 \%$ formalin and stained with $0.1 \%$ crystal violet solution. Cells on the upper side of the membrane were removed using a cotton swab. Images of the invaded or migrated cells were taken in triplicate, and the number of cells was counted in five randomly selected fields under a light microscope (x200 magnification; Olympus IX53; Olympus Corporation, Tokyo, Japan) for statistical analysis.
Western blot analysis. The primary antibodies used in the present study, rabbit polyclonal anti-PTEN (1:500; cat. no. BS6493 and rabbit polyclonal anti- $\beta$-actin (1:500; cat. no. AP0733), were purchased from Bioworld Technology, Inc. (St. Louis Park, MN, USA). Total protein was extracted from the cells using radioimmunoprecipitation assay lysis buffer (Beyotime Institute of Biotechnology, Haimen, China), and protein concentration in the resulting lysates was determined using the bicinchoninic acid protein assay kit (Beyotime Institute of Biotechnology). Equal amounts of protein $(20 \mu \mathrm{g})$ were separated by $10 \%$ sodium dodecyl sulfate-polyacrylamide gel electrophoresis and transferred to polyvinylidene difluoride membranes. After blocking with 5\% nonfat milk in Tris-buffered saline containing 0.1\% Tween-20 (TBST), the membranes were probed with the appropriate primary antibodies overnight at $4{ }^{\circ} \mathrm{C}$, followed by incubation with the goat-anti-rabbit peroxidase-conjugated secondary antibody (1:1,000; Bioworld Technology, Inc.; cat. no. BS13278) in TBST for $1 \mathrm{~h}$ at room temperature. The blots were visualized using enhanced chemiluminescence solution (Pierce Biotechnology, Inc., Rockford, IL, USA) and images were captured and analyzed using the FluorChem imaging system (ProteinSimple, San Jose, CA, USA). $\beta$-actin was used as a loading control.

Luciferase assay. Luciferase reporter plasmids, PGL3-PTEN-3'-UTR WT and PGL3-PTEN-3'-UTR Mut, were purchased from Shanghai GenePharma Co., Ltd. The 786-O and A498 cells were transfected with $0.5 \mu \mathrm{g}$ luciferase reporter plasmids and either $40 \mathrm{nmol}$ miR-22 or NC mimics using Lipofectamine 2000, according to manufacturer's 

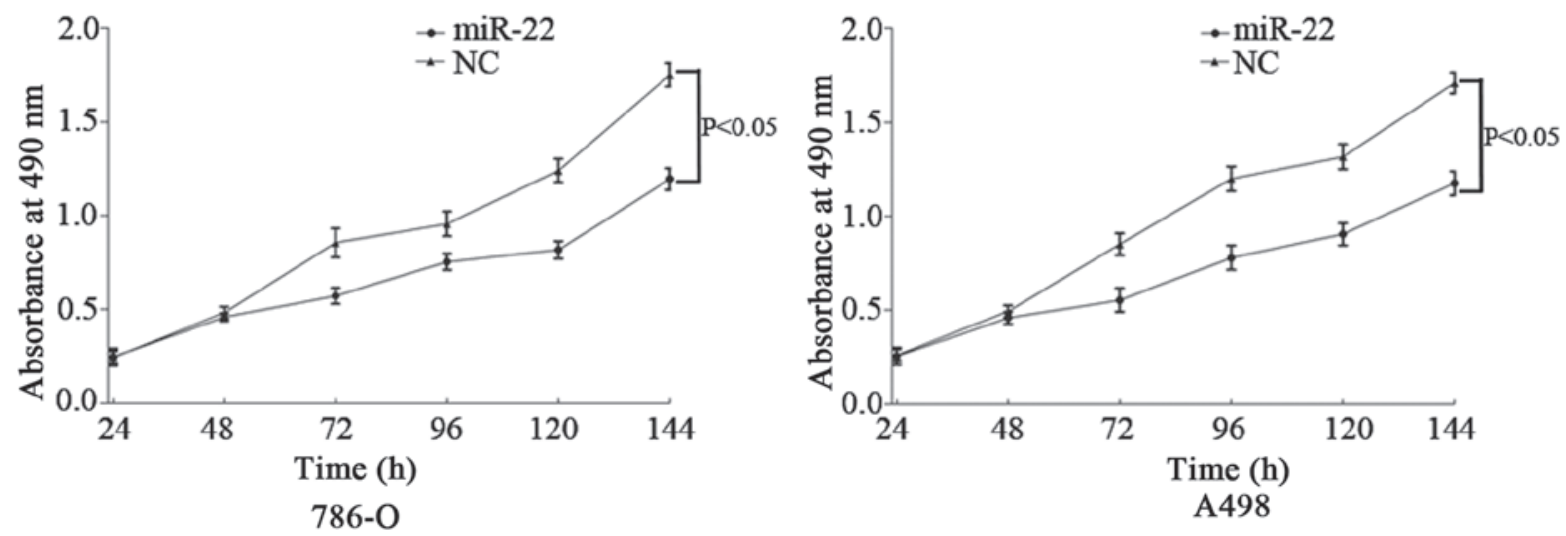

Figure 2. Cell proliferation was determined using the 3-(4,5-dimethylthiazol-2-yl)-2,5-diphenyltetrazolium bromide (MTT) assay. MTT assay revealed that overexpression of microRNA (miR)-22 significantly inhibited cell proliferation in 786-O and A498 renal cell carcinoma cells. Data are presented as the mean \pm standard deviation. NC, negative control.
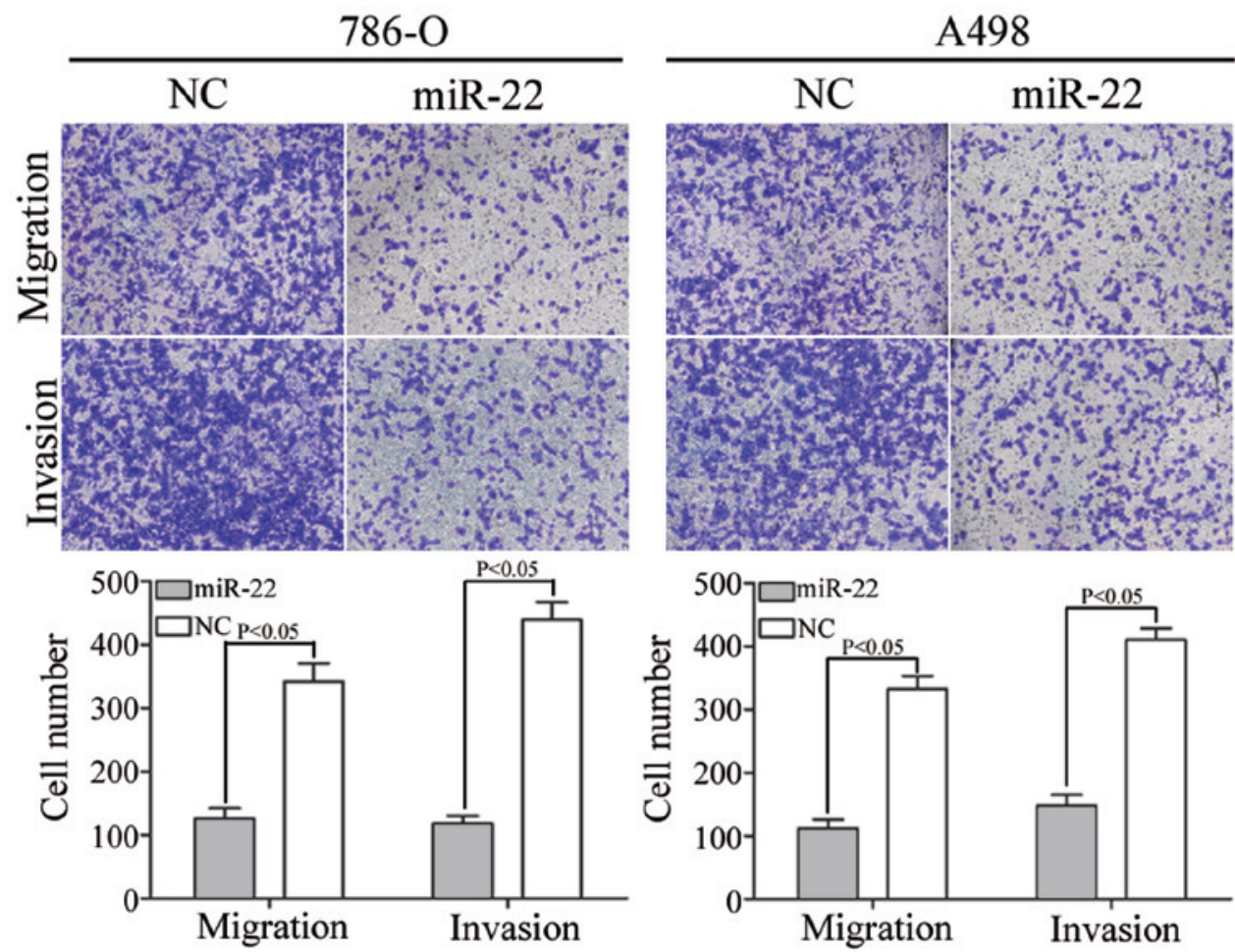

Figure 3. MicroRNA (miR)-22 inhibited cell migration and invasion of 786-O and A498 renal cell carcinoma cells. Following a $12 \mathrm{~h}$ incubation, the number of 786-O and A498 cells that traversed the Transwell membrane was markedly decreased post-transfection with miR-22 mimics. Subsequent to a 24 h incubation, the number of 786-O and A498 cells that traversed the Matrigel-precoated Transwell membrane was markedly decreased post-transfection with miR-22. Magnification, x200. NC, negative control.

protocol. Firefly and Renilla luciferase activity was detected in the cell lysates using the Dual-Luciferase Reporter Assay system (Promega GmbH, Mannheim, Germany) $48 \mathrm{~h}$ post-transfection. Each reporter plasmid was transfected at least three times, on different days, and each sample was assayed in triplicate. Firefly luciferase activity was normalized to Renilla luciferase activity for each transfected well, and measured using an $\mathrm{xMark}^{\mathrm{TM}}$ microplate absorbance spectrophotometer (Bio-Rad Laboratories, Inc.).

Statistical analysis. Data are presented as the mean \pm standard deviation, and were compared using the Student's t-test. The associations between miR-22 expression level and clinicopathological features were analyzed using the Pearson's $\mathrm{X}^{2}$ test. SPSS 16.0 statistical software (SPSS, Inc., Chicago, IL, USA) was used to analyze the data. $\mathrm{P}<0.05$ (two-tailed) was considered to indicate a statistically significant difference.

\section{Results}

miR-22 expression in RCC tissues and its association with clinicopathological factors. A total of 68 RCC tissue samples were analyzed in the present study. As shown in Fig. 1, miR-22 was significantly downregulated in RCC tissues compared 


\section{A}
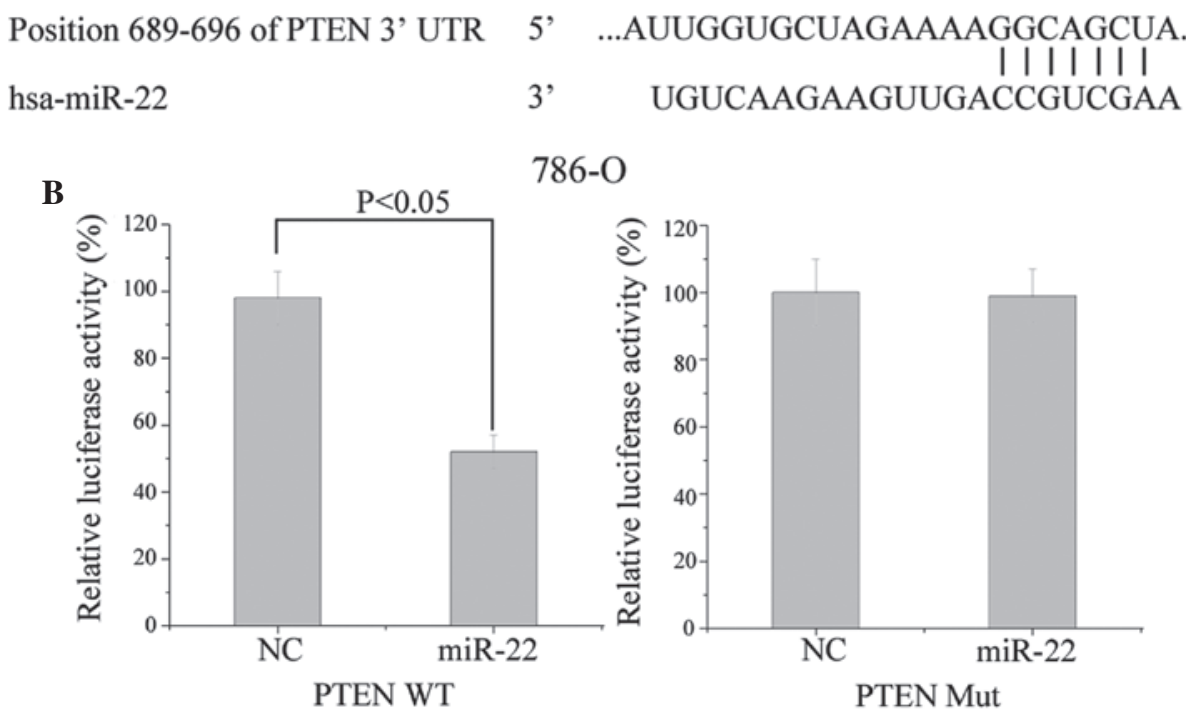

A498
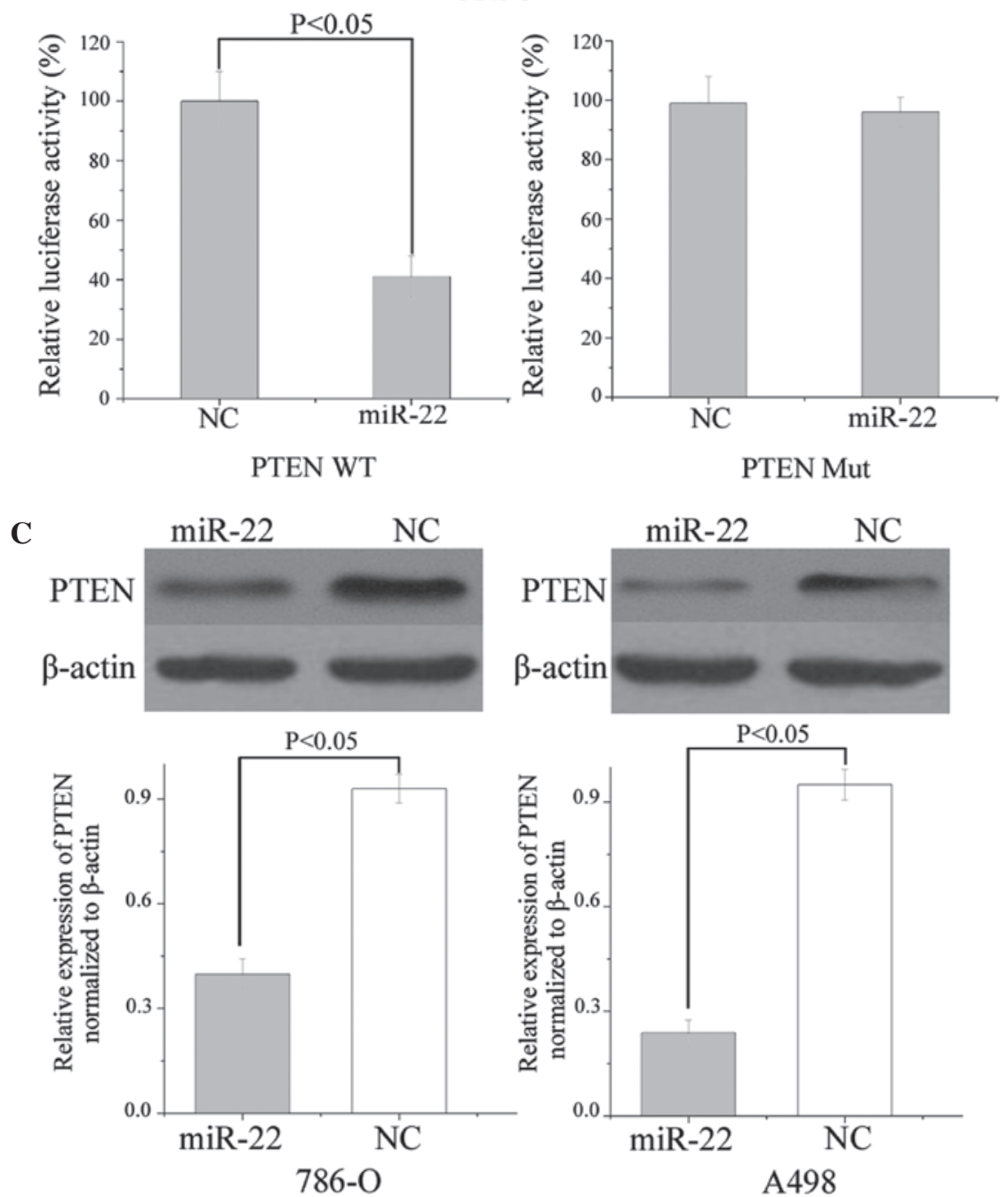

Figure 4. (A) TargetScan was used to identify target genes, and indicated that phosphatase and tensin homolog (PTEN) mRNA contained a microRNA (miR)-22 seven-nucleotide seed match at position 689-696 in the 3'-untranslated region (UTR). (B) PTEN may be a direct target of miR-22 in vitro. Overexpression of miR-22 significantly inhibited the wild-type (WT) but not the mutant (Mut) luciferase activity of PTEN in 786-O and A498 renal cell carcinoma cells. (C) PTEN was significantly downregulated in 786-O and A498 cells post-transfection with miR-22. Data are presented as the mean \pm standard deviation. NC, negative control.

with in NATs $(\mathrm{P}<0.05)$. These results indicate that miR-22 may have an important role in RCC.
The present study also examined whether the expression levels of miR-22 were associated with gender, age, tumor 
diameter, histological grade, tumor stage and lymph node metastasis. Patients with RCC were divided into high or low groups according to the mean expression levels of miR-22. Statistical analysis indicated that miR-22 expression was significantly associated with histological grade, tumor stage and lymph node metastasis (Table I). However, no correlation was detected between miR-22 expression and other clinicopathological factors, including gender, age and tumor diameter.

miR-22 suppresses cell proliferation in 786-O and A498 RCC cells. To verify the effects of miR-22 on cell proliferation, an MTT assay was conducted. As shown in Fig. 2, upregulation of miR-22 significantly inhibited cell proliferation in 786-O and A498 RCC cells. The MTT assay revealed that $144 \mathrm{~h}$ post-transfection, the suppressive rate of miR-22 on cell proliferation reached $29.71 \pm 5.1 \%$ in $786-\mathrm{O}$ cells and $31.21 \pm 4.7 \%$ in A498 cells $(\mathrm{P}<0.05)$.

miR-22 suppresses cell migration and invasion of 786-O and A498 RCC cells. To determine the effects of miR-22 on tumor cell migration and invasion, a Transwell assay was conducted. As shown in Fig. 3, the migratory and invasive ability of miR-22-transfected RCC cells was markedly decreased, as compared with the NC mimic-transfected cells $(\mathrm{P}<0.05)$. These results indicate that miR-22 may suppress the migration and invasion of 786-O and A498 RCC cells.

PTEN is a direct target gene of $\mathrm{miR}-22$ in 786-O and A498 RCC cells. To identify target genes of miR-22 in RCC, a public database (TargetScan; http://www.targetscan.org) was used. As shown in Fig. 4A, PTEN was predicted to be a target of miR-22. To verify whether miR-22 directly targeted PTEN, luciferase reporter assays were conducted. As shown in Fig. 4B, miR-22 significantly inhibited the wild-type $(\mathrm{P}<0.05)$ but not the mutant luciferase activity of PTEN in 786-O and A498 RCC cells.

Western blotting was also conducted to determine whether PTEN was downregulated post-transfection of 786-O and A498 RCC cells with miR-22 mimics. As shown in Fig. 4C, PTEN was significantly downregulated in RCC cells post-transfection with miR-22 $(\mathrm{P}<0.05)$. These results indicate that PTEN may be a direct target gene of miR-22 in 786-O and A498 RCC cells.

\section{Discussion}

The expression of miR-22 has been reported to be altered in certain types of cancer. In colon cancer, Li et al (16) demonstrated that miR-22 was downregulated, and ectopic expression of miR-22 decreased cell migration and invasion by directly targeting matrix metalloproteinase (MMP)-2 and MMP-9. In gastric cancer, Guo et al (17) demonstrated that miR-22 was downregulated, and its overexpression inhibited cell migration and invasion by inhibiting the expression of Sp1 transcription factor. In addition, Wang et al (22) reported that miR-22 suppressed gastric cancer cell proliferation and invasion by inhibiting CD151. In ovarian cancer, miR-22 was verified as a potential metastatic inhibitor; there was a negative correlation between miR-22 expression and metastatic potential in ovarian cancer cells (18). In hepatocellular carcinoma, the expression levels of miR-22 were decreased, and downregulation of miR-22 was predictive of poor survival in patients. Furthermore, overexpression of miR-22 significantly inhibited the proliferation of hepatocellular carcinoma cells (19). In lung cancer, Ling et al (20) revealed that miR-22 was weakly expressed and suppressed cancer cell progression via post-transcriptional regulation of Erb-B2 receptor tyrosine kinase 3 . The present study expanded our knowledge regarding the expression and function of miR-22 in cancer.

The present study was the first, to the best of our knowledge, to provide evidence that miR-22 was downregulated in human RCC. Therefore, it may be hypothesized that miR-22 exerts a tumor suppressive role in RCC development and progression. Notably, overexpression of miR-22 in RCC cell lines reduced cell proliferation, migration and invasion, thus suggesting a tumor suppressive role of miR-22. These results may have clinical implications in the future.

An important molecular link was identified between miR-22 and PTEN in the present study. TargetScan predicted that PTEN was a direct target gene of miR-22. The 3'-untranslated region (UTR) of PTEN mRNA was shown to contain a miR-22 seven-nucleotide seed match at position 689-696. Furthermore, the results of the luciferase activity assay indicated that miR-22 directly targeted the PTEN 3'-UTR, as predicted by bioinformatics. miR-22 upregulation also led to downregulation of PTEN protein in RCC cell lines. These findings revealed that miR-22 may regulate PTEN expression in vitro, and may have a tumor suppressive role in RCC development and progression.

PTEN, which is one of the most studied tumor suppressor genes, is located on human chromosome 10q23 and contains nine exons encoding a 403-amino acid protein, which is predominantly cytoplasmic $(23,24)$. PTEN suppresses activation of the Ras/mitogen-activated protein kinase pathway via inhibition of Src-homology collagen phosphorylation, following epidermal growth factor stimulation. This suppression has been provided as an explanation for the tumor suppressive effects of PTEN (25). PTEN is frequently deleted or mutated in various types of human carcinomas, thus suggesting a crucial role in tumor development (26). Deletion or mutation of PTEN contributes toward uncontrolled AKT activation, which results in tumor development and progression via the suppression of cell apoptosis (27). Mechanisms underlying PTEN inactivation include mutations, deletions and promoter hypermethylation (28). The results of the present study suggested that miR-22 may suppress RCC cell proliferation, migration and invasion by directly targeting PTEN, thus suggesting that miR-22 may be investigated as a target for the treatment of in RCC.

In conclusion, the present study is the first, to the best of our knowledge, to indicate that miR-22 is downregulated in RCC tissues, and may contribute toward cell proliferation, migration and invasion via downregulation of PTEN in RCC cell lines. miRNA-based therapy is expected to be more efficient than the traditional single target therapy, since miRNAs regulate several target genes simultaneously. The present study provided a novel therapeutic target, the miR-22/PTEN axis, in RCC. The findings of the present study may be beneficial for the development of an effective therapy against RCC. 
Future work is required to address whether the potential of miR-22 may be fully realized in cancer treatment. If so, it may be beneficial for the treatment of RCC.

\section{References}

1. White NM and Yousef GM: MicroRNAs: Exploring a new dimension in the pathogenesis of kidney cancer. BMC Med 8: $65,2010$.

2. Saini S, Yamamura S, Majid S, Shahryari V, Hirata H, Tanaka Y and Dahiya R: MicroRNA-708 induces apoptosis and suppresses tumorigenicity in renal cancer cells. Cancer Res 71: 6208-6219, 2011.

3. Lian JH, Wang WH, Wang JQ, Zhang YH and Li Y: MicroRNA-122 promotes proliferation, invasion and migration of renal cell carcinoma cells through the PI3K/Akt signaling pathway. Asian Pac J Cancer Prev 14: 5017-5021, 2013.

4. Yu ZH, Zhang Q, Wang YD, Chen J, Jiang ZM, Shi M, Guo X, Qin J, Cui GH, Cai ZM, et al: Overexpression of cyclooxygenase-1 correlates with poor prognosis in renal cell carcinoma. Asian Pac J Cancer Prev 14: 3729-3734, 2013.

5. Jiang Z, Chu PG, Woda BA, Liu Q, Balaji KC, Rock KL and Wu CL: Combination of quantitative IMP3 and tumor stage: A new system to predict metastasis for patients with localized renal cell carcinomas. Clin Cancer Res 14: 5579-5584, 2008.

6. Wu D, Li M, Wang L, Zhou Y, Zhou J, Pan H and Qu P MicroRNA-145 inhibits cell proliferation, migration and invasion by targeting matrix metallopeptidase-11 in renal cell carcinoma. Mol Med Rep 10: 393-398, 2014.

7. Li M, Wang Y, Song Y, Bu R, Yin B, Fei X, Guo Q and Wu B: MicroRNAs in renal cell carcinoma: A systematic review of clinical implications (Review). Oncol Rep 33: 1571-1578, 2015.

8. Bartel DP: MicroRNAs: Genomics, biogenesis, mechanism, and function. Cell 116: 281-297, 2004.

9. Dang Y,Zhao S, Qin Y,Han T,LiW and ChenZJ: MicroRNA-22-3p is down-regulated in the plasma of Han Chinese patients with premature ovarian failure. Fertil Steril 103: 802-807, 2015.

10. Huang ZP and Wang DZ: MiR-22 in cardiac remodeling and disease. Trends Cardiovasc Med 24: 267-272, 2014.

11. Bartel DP: MicroRNAs: Target recognition and regulatory functions. Cell 136: 215-233, 2009.

12. Friedman RC, Farh KK, Burge CB and Bartel DP: Most mammalian mRNAs are conserved targets of microRNAs. Genome Res 19: 92-105, 2009

13. Kwak PB, Iwasaki S and Tomari Y: The microRNA pathway and cancer. Cancer Sci 101: 2309-2315, 2010.

14. Chen X, Bo L, Zhao X and Chen Q: MicroRNA-133a inhibits cell proliferation, colony formation ability, migration and invasion by targeting matrix metallopeptidase 9 in hepatocellular carcinoma. Mol Med Rep 11: 3900-3907, 2015.
15. Eacker SM, Dawson TM and Dawson VL: Understanding microRNAs in neurodegeneration. Nat Rev Neurosci 10: 837-841, 2009

16. Li B, Song Y, Liu TJ, Cui YB, Jiang Y, Xie ZS and Xie SL: MiRNA-22 suppresses colon cancer cell migration and invasion by inhibiting the expression of T-cell lymphoma invasion and metastasis 1 and matrix metalloproteinases 2 and 9. Oncol Rep 29: 1932-1938, 2013.

17. Guo MM, Hu LH, Wang YQ, Chen P, Huang JG, Lu N, He JH and Liao CG: MiR-22 is down-regulated in gastric cancer, and its overexpression inhibits cell migration and invasion via targeting transcription factor Sp1. Med Oncol 30: 542, 2013.

18. Li J, Liang S, Yu H, Zhang J, Ma D and Lu X: An inhibitory effect of miR-22 on cell migration and invasion in ovarian cancer. Gynecol Oncol 119: 543-548, 2010.

19. Zhang J, Yang Y, Yang T, Liu Y, Li A, Fu S, Wu M, Pan Z and Zhou W: MicroRNA-22, downregulated in hepatocellular carcinoma and correlated with prognosis, suppresses cell proliferation and tumourigenicity. Br J Cancer 103: 1215-1220, 2010.

20. Ling B, Wang GX, Long G, Qiu JH and Hu ZL: Tumor suppressor miR-22 suppresses lung cancer cell progression through post-transcriptional regulation of ErbB3. J Cancer Res Clin Oncol 138: 1355-1361, 2012

21. Tao J, Lu Q, Wu D, Li P, Xu B, Qing W, Wang M, Zhang Z and Zhang W: microRNA-21 modulates cell proliferation and sensitivity to doxorubicin in bladder cancer cells. Oncol Rep 25: 1721-1729, 2011.

22. Wang $X$, Yu H, Lu X, Zhang $P$, Wang $M$ and Hu Y: MiR-22 suppresses the proliferation and invasion of gastric cancer cells by inhibiting CD151. Biochem Biophys Res Commun 445 175-179, 2014.

23. Rizvi MM, Alam MS, Mehdi SJ, Ali A and Batra S: Allelic loss of 10q23.3, the PTEN gene locus in cervical carcinoma from northern indian population. Pathol Oncol Res 18: 309-313, 2012.

24. Cheng T, Zhang JG, Cheng YH, Gao ZW and Ren XQ: Relationship between PTEN and Livin expression and malignancy of renal cell carcinomas. Asian Pac J Cancer Prev 13: 2681-2685, 2012.

25. Gu J, Tamura M and Yamada KM: Tumor suppressor PTEN inhibits integrin- and growth factor-mediated mitogen-activated protein (MAP) kinase signaling pathways. J Cell Biol 143: 1375-1383, 1998

26. Brenner W, Färber G, Herget T, Lehr HA, Hengstler JG and Thüroff JW: Loss of tumor suppressor protein PTEN during renal carcinogenesis. Int J Cancer 99: 53-57, 2002.

27. Sun H, Lesche R, Li DM, Liliental J, Zhang H, Gao J, Gavrilova N, Mueller B, Liu X and Wu H: PTEN modulates cell cycle progression and cell survival by regulating phosphatidylinositol 3,4,5,-trisphosphate and Akt/protein kinase B signaling pathway. Proc Natl Acad Sci USA 96: 6199-6204, 1999.

28. Hager M, Haufe H, Kemmerling R, Mikuz G, Kolbitsch C and Moser PL: PTEN expression in renal cell carcinoma and oncocytoma and prognosis. Pathology 39: 482-485, 2007. 